\title{
Light Curves of Planetary Transits: How About Ellipticity?
}

\author{
Carolina von Essen, Klaus F. Huber and Jürgen H. M. M. Schmitt \\ Hamburger Sternwarte, University of Hamburg, \\ (21029) Hamburg, Germany \\ email: cessen@hs.uni-hamburg.de
}

\begin{abstract}
The observation of transit light curves has become a key technique in the study of exoplanets, since modeling the resulting transit photometry yields a wealth of information on the planetary systems. Considering that the limited accuracy of ground-based photometry does directly translate into uncertainties in the derived model parameters, simplified spherical planet models were appropriate in the past. With the advent of space-based instrumentation capable of providing photometry of unprecedented accuracy, however, a need for more realistic models has arisen.
\end{abstract}

Keywords. exoplanets, light curves, modelling, oblateness

\section{Motivation}

The gas giants in our Solar System are not spherical but oblate. Oblateness, $f$, is defined as $f=\left(R_{e q}-R_{p o l}\right) / R_{e q}$, where $R_{e q}$ corresponds to the equatorial radius and $R_{p o l}$ to the polar radius. Oblateness values for our Solar System gas giants are shown in Table 1.

A relation between oblateness and observables quantities can be obtained by taking into account the Darwin-Radau relation. Defining the parameter $\zeta=\frac{\text { mom. of inertia }}{M R_{e q}^{2}}=$ $\frac{2}{3}\left[1-\frac{2}{5}\left(\frac{5}{2} \frac{q}{f}-1\right)^{\frac{1}{2}}\right]$, where the parameter $q=\frac{\Omega^{2} R_{e q}^{3}}{G M_{P l}}$ is the ratio between the centripetal and gravitational acceleration, defined by Barnes and Fortney (2003), we obtain the following expression for the oblateness: $f=\frac{4 \pi^{2} R^{3}}{G M P^{2}}\left[\frac{5}{2}\left(1-\frac{3}{2} \zeta\right)^{2}+\frac{2}{5}\right]^{-1}$. Assuming $\zeta \sim 0.25$ (in analogy to gas giants in the Solar System) and tidal locking, we calculated $f$ for different exoplanets (see Table 2).

\section{Analytical model: comparing oblate and spherical planets}

We assume that the planet shape can be modelled as a rotational ellipsoid and the star as a sphere, and the projections are an ellipse and a circle, respectively. In the case $i=90^{\circ}$, the symmetry of the problem allows an analytical model for the flux drop during primary transit. The difference between the models (Fig. 1) can be resolved for instance by the Kepler Telescope, given a good model for limb darkening.

Table 1. Oblateness values for the giant planets in our Solar System.

\begin{tabular}{|c|c|c|c||c|c|c|c|}
\hline Planet Name & $R_{e q}$ & $R_{\text {pol }}$ & $f$ & Planet Name & $R_{e q}$ & $R_{\text {pol }}$ & $f$ \\
\hline Jupiter & $71492 \mathrm{~km}$ & $66854 \mathrm{~km}$ & 0.0648 & Uranus & $25559 \mathrm{~km}$ & $24973 \mathrm{~km}$ & 0.0229 \\
Saturn & $60268 \mathrm{~km}$ & $54364 \mathrm{~km}$ & 0.0979 & Neptune & $24764 \mathrm{~km}$ & $24341 \mathrm{~km}$ & 0.0171 \\
\hline
\end{tabular}


Table 2. Oblateness values for short period exoplanets.

\begin{tabular}{|c|c|c|c|c|c|c|c|}
\hline System & $\mathrm{P}[\mathrm{d}]$ & $\mid<\rho>(\operatorname{cgs})$ & $f$ & System & $\mathrm{P}[\mathrm{d}]$ & $\mid<\rho>(\mathrm{cgs})$ & $f$ \\
\hline HD 149026 & $\sim 2.875$ & $\sim 1.17$ & 0.00152 & WASP - 15 & $\sim 3.752$ & $\sim 0.247$ & 0.00422 \\
\hline HD 189733 & $\sim 2.218$ & $\sim 0.91$ & 0.00328 & CoRoT - 1 & $\sim 1.508$ & $\sim 0.38$ & 0.017 \\
\hline HD 209458 & $\sim 3.524$ & $\sim 0.38$ & 0.00365 & WASP - 19 & $\sim 0.788$ & $\sim 0.486$ & 0.048 \\
\hline
\end{tabular}
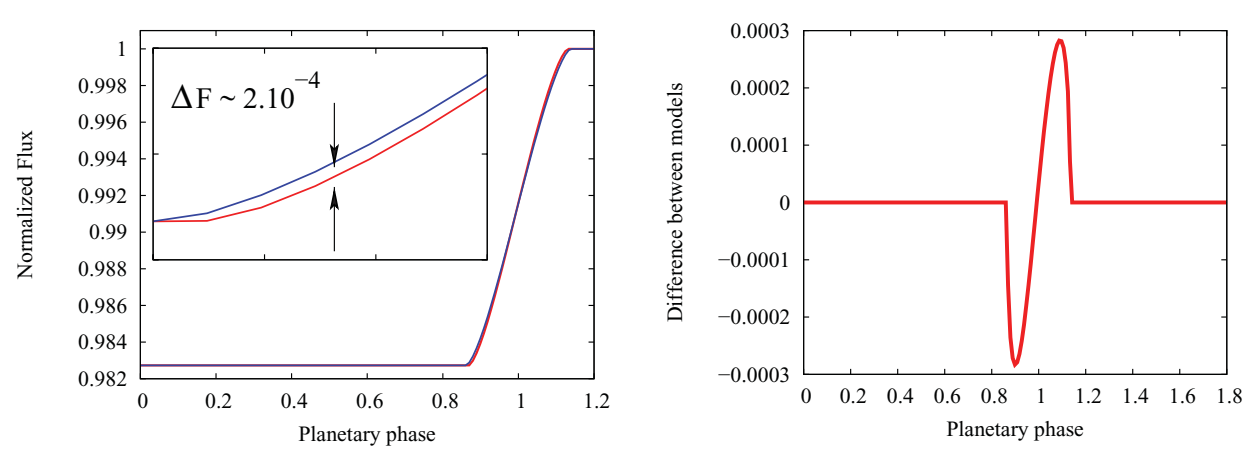

Figure 1. Left: Difference between the two analytical models, considering oblate and spherical planets. Right: Oblateness effects are important only during ingress and egress.

\section{Numerical model: Comparison to Kepler data}

A realistic model must include an arbitrary inclination and limb darkening, and it has to be compared to real data. We find that models with vanishing and large oblateness fit the data equally well, albeit with different fit parameters. However, one has to take into account that oblateness and inclination are degenerate; an ideal method would be the determination of oblateness and inclination independently. A possible way to minimize the degeneration effects is to take into account the mass - radius relationship for the host-star, such as $R=1.24 M^{0.67}$ (for $\mathrm{M}>1.3 M_{\odot}$ ). Fig. 2 shows that the inferred mass of the host star depends on the adopted planetary oblateness. Since KIC 9941662 is of spectral type A3, one clearly favors a low oblateness.
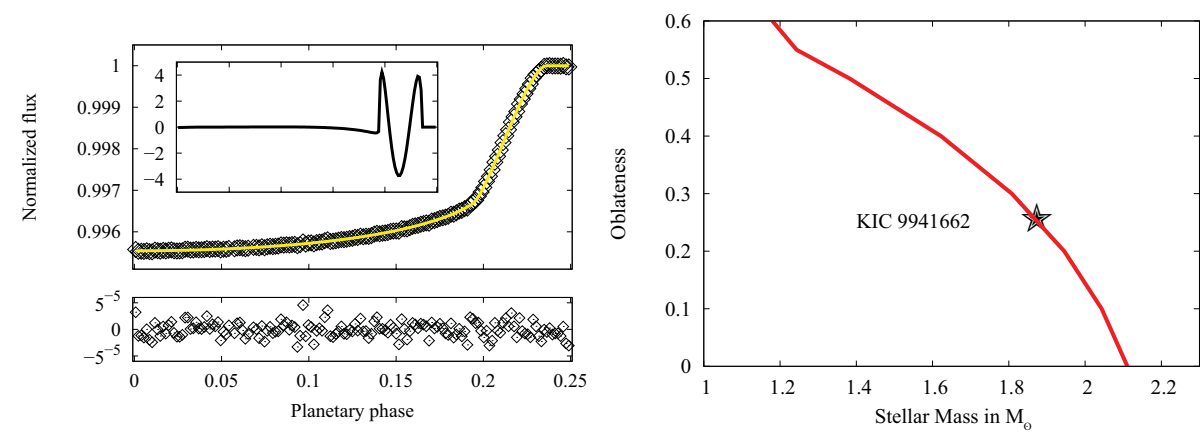

Figure 2. Left: Kepler light curve of KIC 9941662 and fit considering $f=0.5$ for $\chi^{2}$ minimization. Small box: Difference between two models $(\mathrm{f}=0$ and $\mathrm{f}=0.5)$, multiplied by $10^{6}$. Right: Host-star mass-oblateness relationship for the case of KIC 9941662. ST $\sim \mathrm{A} 3, T_{\text {eff }} \sim 8800 \mathrm{~K}$.

\section{References}

Mandel, K. \& Agol, E. 2002, ApJ, 580, 171

Barnes, J. W. \& Fortney, J. J. 2003, ApJ, 588, 545

Carter, J. A. \& Winn, J. N. 2010, ApJ, 716, 850 